\title{
First Results from the CMD-3 Detector at the VEPP-2000 $\mathrm{e}^{+} \mathrm{e}^{-}$Collider
}

\section{Simon EIDELMAN for the CMD-3 Collaboration*}

Budker Institute of Nuclear Physics RAS, Novosibirsk, Russia

E-mail: eidelman@inp.nsk.su

The CMD-3 detector at the VEPP-2000 electron-positron collider in the Budker Institute in Novosibirsk collected about $20 \mathrm{pb}^{-1}$ in the center-of-mass energy range from 1 to $2 \mathrm{GeV}$ during the first year of operation. First results on the hadronic cross sections are reported important for improving the precision of the theoretical predictions for the muon anomalous magnetic moment.

The 2011 Europhysics Conference on High Energy Physics-HEP 2011,

July 21-27, 2011

Grenoble, Rhône-Alpes France

${ }^{*}$ Speaker. 


\section{Introduction}

Since 2009 the VEPP-2000 $e^{+} e^{-}$collider has been running in the Budker Institute of Nuclear Physics in Novosibirsk. VEPP-2000 is an upgrade of the previous VEPP-2M collider, which should cover the center-of-mass (c.m.) energy range from threshold of hadron production to $2 \mathrm{GeV}$ and a maximum luminosity of $10^{32} \mathrm{~cm}^{-2} \mathrm{~s}^{-1}$ [1]. VEPP-2000 delivers luminosity to two detectors CMD-3 [2] and SND [3].

The main goal of experiments at VEPP-2000 is a measurement of the total and exclusive cross sections of $e^{+} e^{-} \rightarrow$ hadrons with high precision reaching better than $1 \%$ for $e^{+} e^{-} \rightarrow \pi^{+} \pi^{-}$and $2-3 \%$ for multhadronic final states. Such measurements will provide valuable information about interactions of light quarks which could be extracted from data by studying spectroscopy of light vector mesons and their excitations, investigation of mesons with various $J^{P C}$, production of $p \bar{p}$ and $n \bar{n}$ pairs near threshold and searches for various exotics.

High-precision measurements of low energy cross sections also have numerous implications for various fundamental quantities, such as muon anomalous magnetic moment, $a_{\mu}$, where a more than $3.5 \sigma$ deviation is observed from the Standard Model predictions [4], the running $\alpha$ [4], $m_{u(d)}$ and quark/gluon condensates from QCD sum rules [5], tests of CVC by comparing $e^{+} e^{-}$and $\tau$ [6], etc.

\section{Experiment}

The VEPP-2000 accelerator complex is shown in Fig. 1. A peak luminosity of $2 \times 10^{31} \mathrm{~cm}^{-2} \mathrm{~s}^{-1}$ has already been achieved using a scheme of round beams. The design luminosity will be hopefully achieved in 2012 after the new injection complex is commissioned. In 2010-2011 two scans of the c.m. range between 1 and $2 \mathrm{GeV}$ were performed (up and down).with a total integrated luminosity of about $22 \mathrm{pb}^{-1}$. There was also a brief scan of the $\phi$ meson energy with a goal to determine the correctness of the collider energy scale and a few runs just above the threshold of proton-antiproton pair production.

\section{Layout of the VEPP-2000 complex}

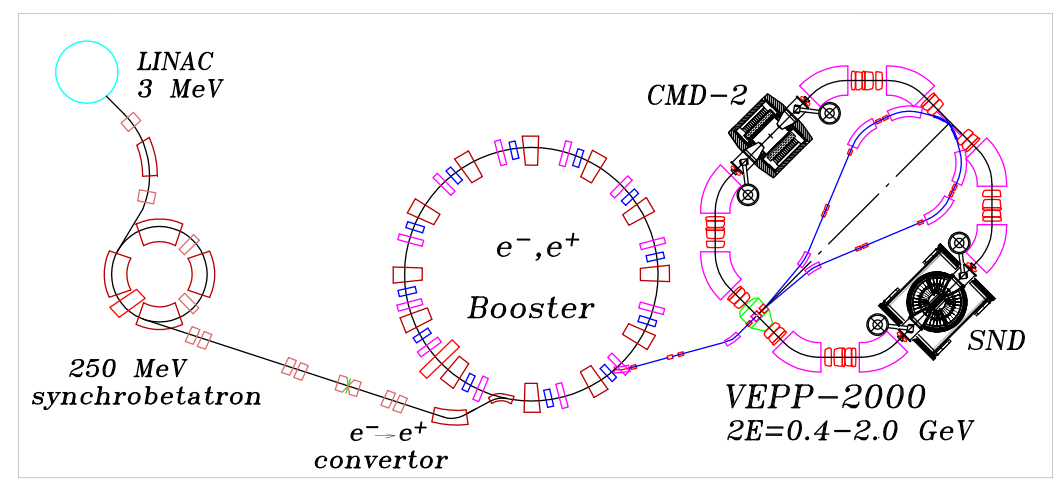

Figure 1: The lay-out of the VEPP-2000 complex

The CMD-2 detector was upgraded to meet new conditions of data taking. A new detector, dubbed the CMD-3, is shown in Fig. 2. 

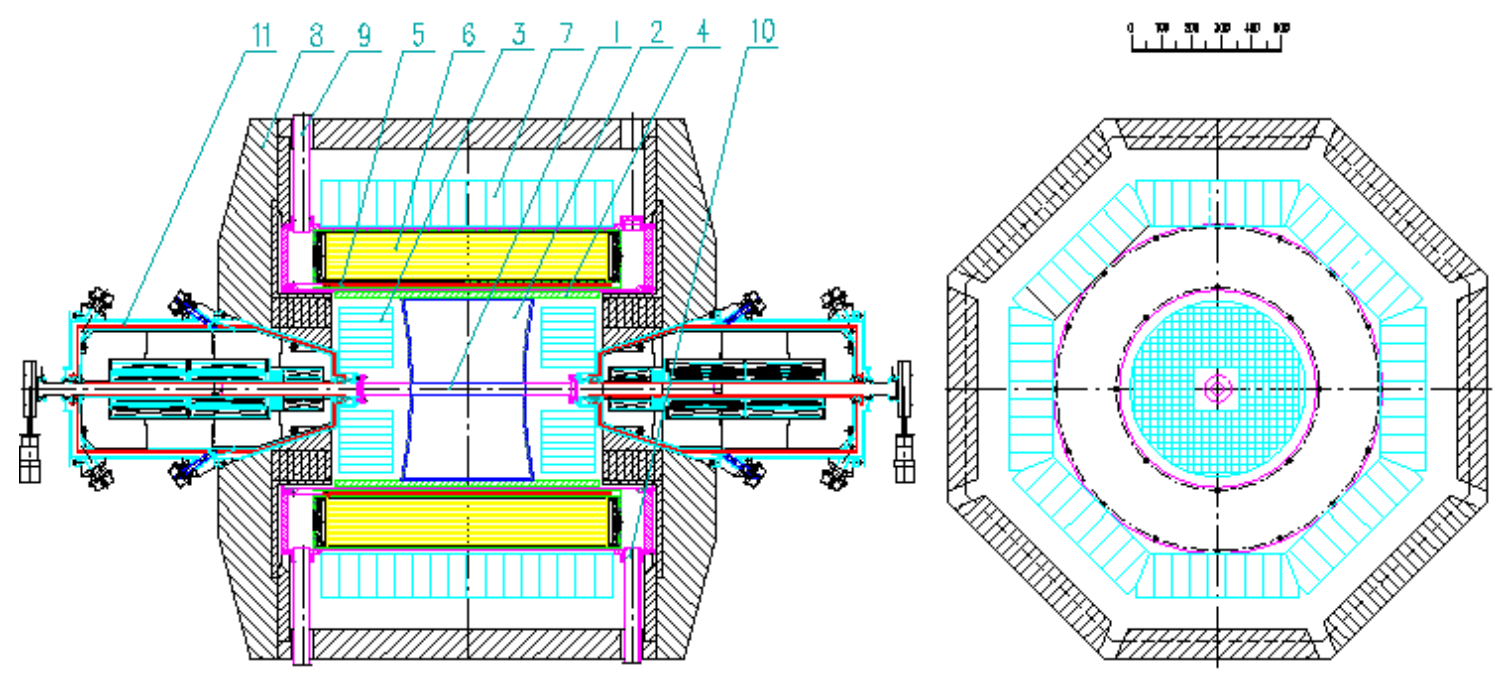

Figure 2: CMD3 detector: 1 Beam pipe, 2 Drift chamber, 3 BGO calorimeter, 4 Z chamber, 5 SC solenoid, 6 LXe calorimeter, 7 CsI calorimeter, 8 Flux return, 9 LHe supply, 10 Vacuum pumpdown, 11 SC focusing magnets.

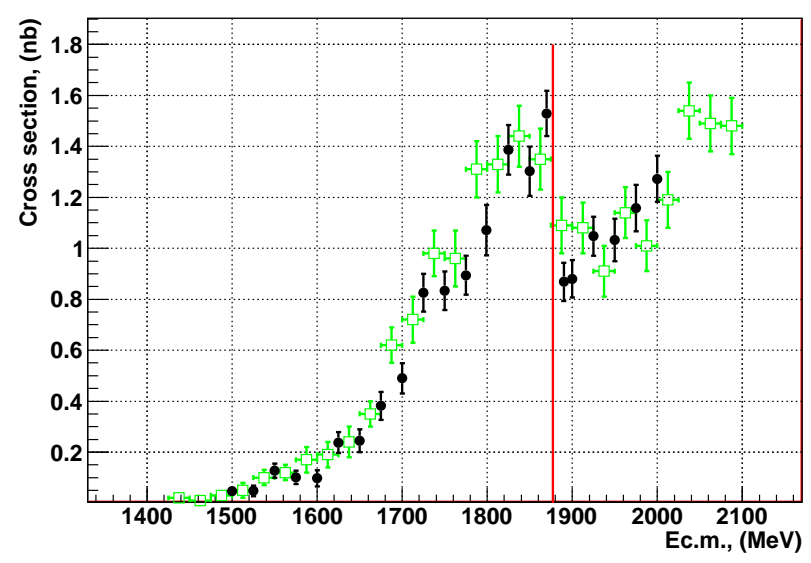

Figure 3: Cross section of $e^{+} e^{-} \rightarrow 3 \pi^{+} 3 \pi^{-}$: open squares - BaBar, black circles - CMD-3

\section{Results}

Many final multihadron states have already been detected and preliminary values of the cross sections of their productions obtained. We show in Fig. 3 the cross section of the process $e^{+} e^{-} \rightarrow$ $3 \pi^{+} 3 \pi^{-}$in comparison with the results of the $\mathrm{BaBar}$ experiment [7]. The measurement of $\mathrm{BaBar}$ was performed using the method of radiative return and confirmed the old results, which revealed a dip in the cross section near $1900 \mathrm{MeV}$ [8]. Our results shown by black circles in Fig. 3 are consistent with those of BaBar. The nature of the dip is still unknown: it can be due to a new $\rho(1900)$ state, interference of the $\rho(1700)$ with the physical $3 \pi^{+} 3 \pi^{-}$background or, taking into account a close $p \bar{p}$ threshold, a new baryonium state.

Finally, it is worth noting that CMD-3 reliably identifies events of the process $e^{+} e^{-} \rightarrow p \bar{p}$ 


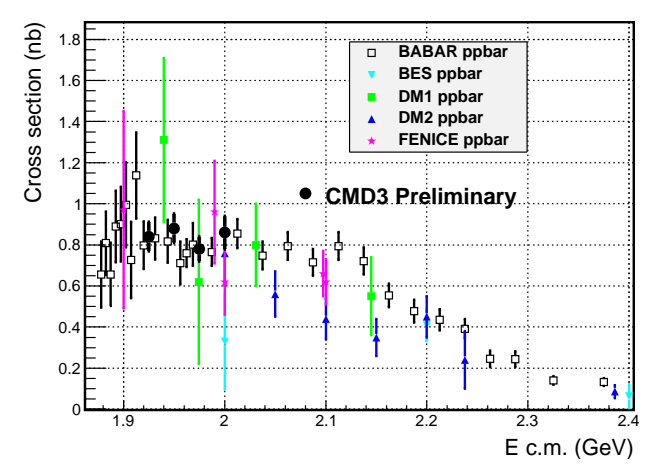

Figure 4: Cross section of $e^{+} e^{-} \rightarrow p \bar{p}$ : black squares - BaBar, blue circles - CMD-3

using $\bar{p}$ annihilation in the detector material. The cross section shown in Fig. 4 was measured at 4 energy points and is consistent with the previous measurements, in particular with that of BaBar [9].

\section{Acknowledgments}

We greatly appreciate the efforts of the staff of VEPP-2000 to provide good operation of the complex and the staff of experimental laboratories for the permanent support in preparing and performing this experiment.

This work was partially supported by the Russian Foundation for Basic Research, Grants 09-02-01143, 10-02-00695, 11-02-00112, 11-02-00558, 11-02-91343 and DFG Grant GZ:HA1457/71.

\section{References}

[1] Yu.M. Shatunov et al., Proc. of EPAC 2000, Vienna, Austria, p.439.

[2] V.M. Aulchenko et al., Preprint BINP 2001-45.

[3] P.M. Astigeevich et al.,Preprint BINP 2011-21.

[4] K. Hagiwara et al., J. Phys. G 38, 085003 (2011).

[5] S.I. Eidelman, L.M. Kurdadze and A.I. Vainshtein, Phys. Lett. B 82, 278 (1979).

[6] S.I. Eidelman and V.N. Ivanchenko, Phys. Lett. B 257, 437 (1991).

[7] B. Aubert et al., Phys. Rev. D 73, 052003 (2006).

[8] K. Nakamura et al.,J. Phys. G 37, 075021 (2010).

[9] B. Aubert et al., Phys. Rev. D 73, 012005 (2006). 